\title{
An Efficient way of Number Plate Alphabets and Numbers Extraction for Security Purpose
}

\author{
Muhammad Abid Saeed ${ }^{1}$, Muhammad Waqas ${ }^{1}$, Zeeshan Akbar ${ }^{1}$, Niaz Ali $^{2}$ \\ ${ }^{I}$ Department of Electrical Engineering, Sarhad University of Science and Information Technology, Peshawar, \\ ${ }^{2}$ National Power Control Center, NPCC, Pakistan
}

\begin{abstract}
Extraction of number plate plays a key role in the recognition of vehicles. With the advancement in technology and increase in number of vehicles it is the need of the day to recognize registered and unregistered vehicles. For this purpose, in this paper we propose a system to automatically extract digits and alphabets from the number plate of vehicle and search it in the database for its registration. The proposed approach involves four different processes that include Image smoothing, Edge detection, Image segmentation and data extraction. The result shows that the proposed approach can easily detect and extract data from the number plates. Some of the number plates and its extracted data are shown in this paper. The results can further be implemented on automatic extraction of data from shields, sign etc.
\end{abstract}

\section{Introduction}

Vehicle identification is an important part of intelligent traffic system. Due to increase of vehicles and growth of technology it's important to extract data automatically from the number plate and check for its registration. Automatic extraction of data from the number plate has many real time applications i.e. security, controlling traffic capacity, identification etc.

Number plate identification and extraction is very difficult task in the recognition of vehicle because of noise existence, uneven condition of daylight and dim light [1]. There are two different steps involved in the License Plate Recognition (LPR) [2].

- License Plate Localization

- License Plate Digits Recognition

These two steps play an important role in the License Plate Recognition of a vehicle. There are many methods/algorithms developed for License Plate recognition. [8] and [7] extract the License Plate based on vertical edges using Hough Transform. [9] extract the colour using Neural Network and discriminate character using template matching. [10] Extracted the number plate based on genetic algorithm segmentation.[11] uses optical character recognition techniques for number plate recognition.[12] identified automatic vehicle using template matching and knowledge-guided boundary following. [13] Proposed a technique for extracting characters without preceding information of their location and range in the image. [14] Proposed a method for extracting Korean license plate on the basis of the colour of the plate. [6] Proposed feature based approach for the localization of number plate and its characters.

Since number plate standards changes from place to place [3-6] therefore in this paper we propose a fresh based approach for accurately extracting data of same pixel value from the number plate. The proposed approach is independent of the digits and character variation but depends upon the analysis of the number of connected components. This paper is based on the analysis of connected components, containing four major steps that include, RGB to gray-scale conversion, Image smoothing, Edge detection, Image segmentation and data extraction. As RGB to gray-scale conversion is the key step in every image processing algorithm because it is easier to deal with 1 pixel value compare to 3 pixel values at one point and at the end it give us the same result.

The rest of the paper is described as, section 2 describe the median filter for image smoothing, section 3 describe number plate and characters extraction, section 3.1 describe the experimental results and proposed algorithm and section 4 describe the conclusion of the paper.

Some processing that must be performed on images in order to improve its performance. This includes image smoothing, image segmentation and edge detection. 


\section{Median Filter (3X3)}

This filter is used in order to remove salt and pepper noise from the image after converting it in to gray scale image. This filter provides smoothing of unwanted signal that may not be spontaneous and to evade misrepresentation such as extreme thinning or thickening of objects margins. Hence unwanted components which may be considered as connected components are filtered out. We used $3 \mathrm{X} 3$ masking window.

\section{Segmentation}

Image segmentation is an important process in order to separate feature pixels from the rest of the image. There are a lot of methods to obtain it, but in the proposed paper we use edge detection and connected components labeling to obtain segmentation. Sobel edge detector is used in this paper in order to find the edges. We use Sobel Edge detector and 8connectivity in the segmentation of an image because it is efficient and robust in identifying points in a digital image at which the image intensity changes piercingly or more formally has discontinuities compare to other method.

\section{Number Plate and Characters Extraction}

Number Plate extraction is a key step in the recognition of vehicle. The main aim of this part of the research is to input a vehicle image and automatically extract characters from the number plate region with high proficiency.

The beauty of our paper is that we use the same approach for extraction of number plate from vehicle image and also for extraction of digits and alphabets from the number plate, though its efficiency is not that much good but it's very easy in implementation, fast and simple in understanding.

\section{Proposed algorithm and Experimental Results:}

In this paper we proposed an efficient algorithm of Image segmentation in order to separate feature pixels from the rest of the image. When an image is taken it is then converted into gray scale image (figure 2), the method of gray-scale conversion is adopted to increase the speed of processing since it is easier to deal with 1 pixel value rather than to deal with 3 pixel value at one point. The basic idea behind RGB to gray-scale conversion is performed by maintaining the luminance information and eradicating the hue and saturation as shown in the following equation [15].

$\mathrm{Lu}=0.299 * \mathrm{R}+0.587 * \mathrm{G}+0.114 * \mathrm{~B}$

Sobel edge detector is used then for edge detection (fig. 3).

The mask used for edge detection is

$\mathrm{msk}=\left[\begin{array}{lllll}0 & 0 & 0 & 0 & 0 ; \\ 0 & 1 & 1 & 1 & 0 ; \\ 0 & 1 & 1 & 1 & 0 ; \\ 0 & 1 & 1 & 1 & 0 ; \\ 0 & 0 & 0 & 0 & 0 ;\end{array}\right]$

Although there are other good methods for edge detection but in this paper we used sobel edge detector for its robustness and Smoothing of image to reduce the number of connected components. Based on connected components, the proposed algorithm uses 8 connectivity for calculating connected digits and alphabets in the number plate as shown in fig. 4 .

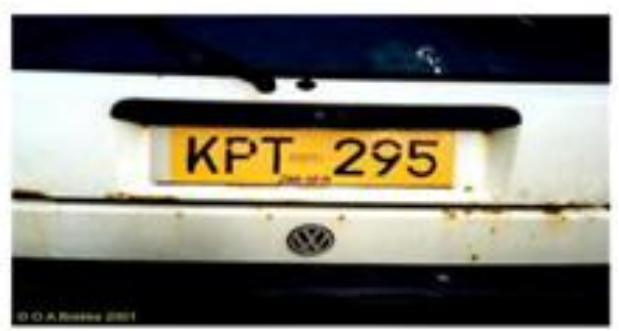

Fig. 1 Back view of a vehicle 


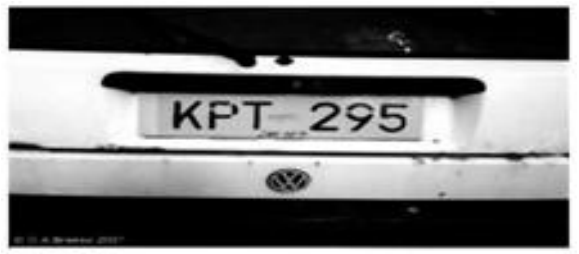

Fig. 2 gray scale conversion

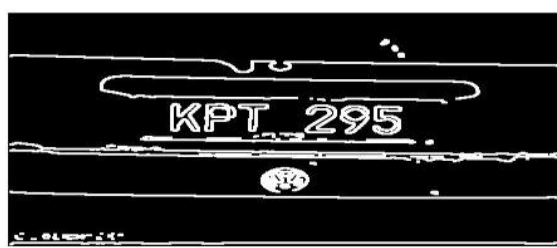

Fig. 3 Edge detection (sobel edge detector)
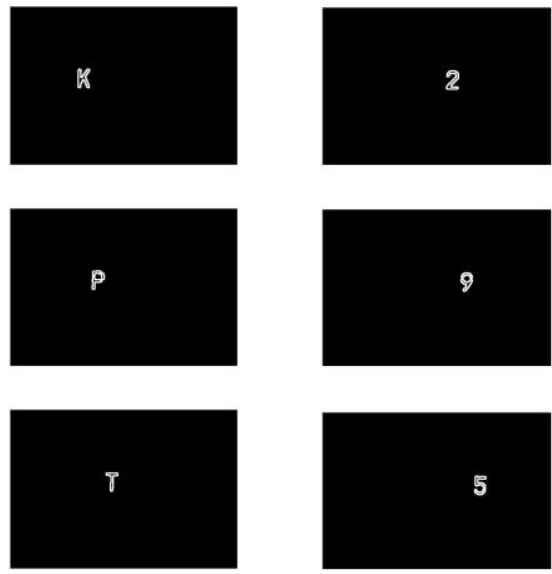

Fig. 4 Character Extraction

Figure 4 shows that characters have been segmented and extracted from the number plate and are shown independently. Since we use 8 connectivity labeling for features/objects extraction. The pixels with the background are tagged 0 . One object is made up from the pixels labeled 1; second object is made up from the pixels labeled 2, and so on. Similarly by passing the desire value from the function using 8connectivity can detect connected components in the number plate and hence extract desire characters/Features. Since each character there in the number plate has its gray level value so by detecting the appropriate gray level value segmentation of character is made [16]. "K", "P", "T", "2", "9", "5" characters has been detected, segmented and extracted from the number plate by pass appropriate values of connected components in the number plate. This is the only disadvantage in the proposed method that one must find the value of connected components that must be passed from the algorithm to find the digits and alphabets in the number plate. Since there is more unknown connected components in an image hence one must pass the desired value for extraction of number plate Character.

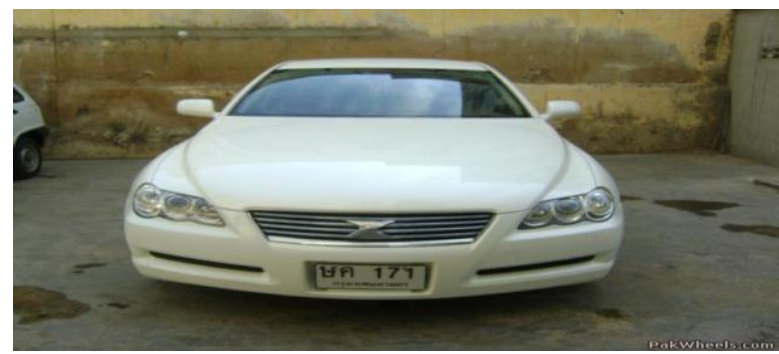

Fig. 5 Front view of a vehicle 


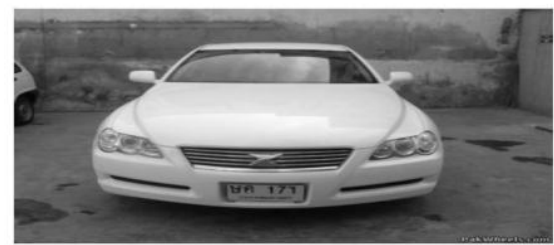

Fig. 6 gray scale conversion

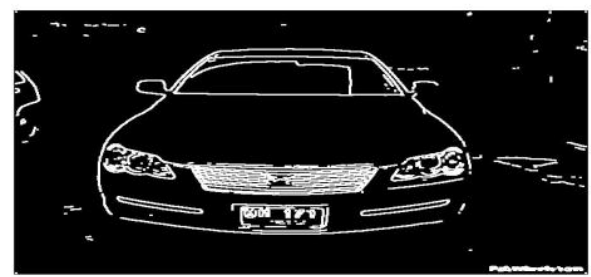

Fig. 7 Edge detection (sobel edge detector)

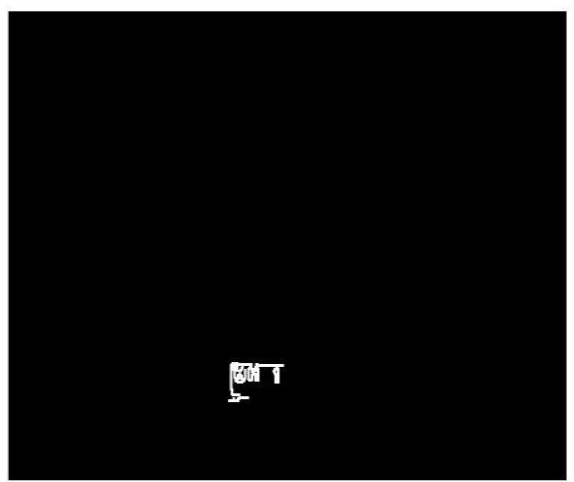

Fig. 8(a) Number Plate and Character Extraction

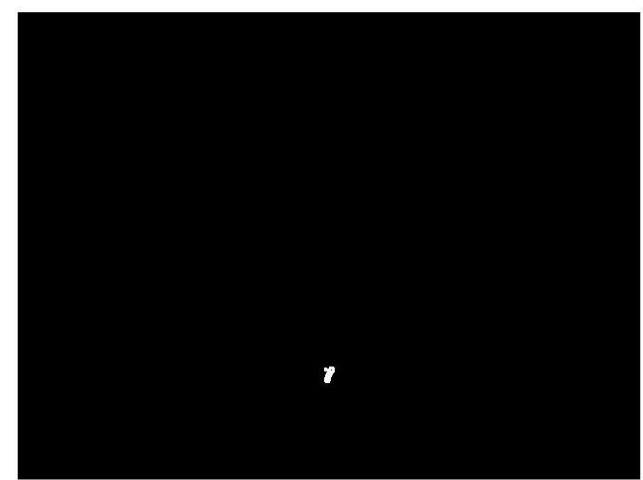

Fig. 8(b) Number Plate and Character Extraction

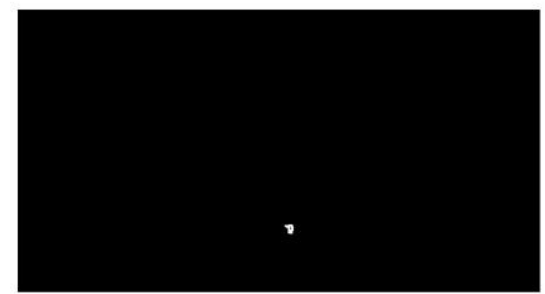

Fig. 8(c) Number Plate and Character Extraction 
The efficiency of our proposed algorithm is good in the extraction of number plate characters and is independent of the language. Since we use 8connectivity, in order to extract characters from the number plate therefore it extracts characters in the form of connected components. Figure 8(a) shows that objects 1,2 and 3 are extracted as single object due to 8-connected labeling, as the proposed algorithm consider these a single object rather than three separate objects. The digits " 7 " and " 1 " are extracted independently as these are considered as single object as shown in figure 8(b) and 8(c) respectively.

\section{Conclusion}

Number plate detection and extraction is a key step towards the safe world. In this paper we have proposed and efficient and new method for automatically extraction of number plates data. This algorithm has been tested on 125 different images and found its accuracy concerning $81 \%$.

The recognition scheme developed in this paper is independent of the Language Alphabets written on number plate, extraction is done with the help of edge detection and connected components labeling. The Experimental results show that the number plates are detected and extracted precisely based on connected components and sobel edge detection algorithm with a success rate of $81 \%$. The complete algorithm in this paper is designed on Matlab (version R2009b) Platform which is cheaper and easily available platform.

\section{References}

[1]. C. Nelson Kennedy Babu, Krishnan Nallaperumal "An Efficient Geometric feature based License Plate Localization and Recognition” International Journal of Imaging Science and Engineering (IJISE) GA,USA,ISSN: 1934-9955, VOL.2,NO.2, APRIL 2008.

[2]. Shyang-Lih Chang, Li-Shien Chen, Yun-Chung Chung, Sei-Wan Chen, "Automatic license plate recognition”, IEEE Transactions on IEEE Transactions on Intelligent Transportation Systems, March 2004, Vol 5,Issue 1, pp: 42 - 53.

[3]. Leonard G. C. Hamey, Colin Priest, "Automatic Number Plate Recognition for Australian Conditions", Proceedings of the Digital Imaging Computing: Techniques and Applications (DICTA), pp. 14- 21, December 2005.

[4]. M. Yu and Y. D. Kim, "An approach to Korean license plate recognition based on vertical edge matching", IEEE International Conference on Systems, Man, and Cybernetics, vol. 4, pp. 2975.2980, 2000.

[5]. M. Sarfraz, M. J. Ahmed and S. A. Ghazi, "Saudi Arabian license plate recognition system", International Conference on Geometric Modeling and Graphics, pp. 36.41, 16-18 July 2003.

[6]. Prathamesh Kulkarni, Ashish Khatri, Prateek Banga, Kushal Shah "A Feature Based Approach for Localization of Indian Number Plates" IEEE 2009

[7]. H.J. Choi, 1987, “A Study on the Extraction and Recognition of a Car Number Plate by Image Processing," Journal of the Korea Institute of Telematics and Electronics, Vo1.24, pp. 309-3 15,1987.

[8]. H.S. Kim, et al., 1991, "Recognition of a Car Number Plate by a Neural Network," Proceedings of the Korea Information Science Society Fall Conference, Vol. 18, pp. 259-262, 1991

[9]. E.R. Lee, P.K. Kim, and H.J. Kim, 1994, "Automatic Recognition of a Car License Plate Using Color Image Processing," Proceedings of the International Conference on Image Processing

[10]. S.K. Kim, D.W. Kim, and H.J. Kim, 1996, “A Recognition of Vehicle License Plate Using a Genetic Algorithm Based Segmentation," Proceedings of 3rd IEEE International Conference on Image Processing,V01.2., pp. 661-664, 1996

[11]. R.A. Lotufo, A.D. Morgan, and AS. Johnson, 1990, “Automatic Number-Plate Recognition,” Proceedings of the IEE Colloquium on Image analysis for Transport Applications, V01.035, pp.6/1-6/6, February 16, 1990.

[12]. A.S. Johnson, B.M. Bird, 1990, "Number-plateMatching for Automatic Vehicle Identification," IEE Colloquium on Electronic Image and Image Processing in Security and Forensic, Aprl, 1990.

[13]. Hontani, H., and Koga, T., (2001), "Character extraction method without prior knowledge on size and information," Proceedings of the IEEE International Vehicle Electronics Conference (IVEC'01), pp. 67-72.

[14]. Park, S. H., Kim, K. I., Jung, K., and Kim, H. J., (1999), “Locating car license plates using neural network," IEE Electronics Letters, vol.35, no. 17, pp. 1475-1477.

[15]. J. Duan, G. Qiu, "Novel Histogram Processing for Colour Image Enhancement", in: Proc. IEEE Int. Conf. Image Graph., 2004, pp. $55-58$.

[16]. Choudhury A. Rahman, Wael Badawy, Ahmad Radmanesh (2003) “A Real Time Vehicle's License Plate Recognition System" Proceedings of the IEEE Conference on Advanced Video and Signal Based Surveillance (AVSS'03) 